\title{
LA INCIDENCIA DE LAS EXTERNALIDADES AMBIENTALES EN LA FORMACIÓN ESPACIAL DE VALORES INMOBILIARIOS: EL CASO DE LA REGIÓN METROPOLITANA DE BARCELONA
}

\section{Jesús Manuel Fitch Osuna ${ }^{1}$}

Centro de Investigación en Arquitectura y Urbanismo

Universidad Autónoma de Nuevo León

\author{
Pilar Garcia Almirall ${ }^{2}$ \\ Centro de Política de Suelo y Valoraciones \\ Universidad Politécnica de Cataluña
}

Remisión articulo: 21-9- 2007

Remisión definitiva: 22 -1-2008

Palabras claves: mercado inmobiliario, precios hedónicos, valoración ambiental, estructura urbana, externalidades ambientales.

\section{Resumen}

Esta investigación ${ }^{3}$ se inscribe en la línea de la valoración ambiental, y se fundamenta en la economía ambiental, urbana y la organización territorial. La hipótesis central es que la población aprecia la calidad ambiental al momento de realizar su elección residencial en función del nivel de ingresos, costumbres y estilo de vida. Para comprobar dicha hipótesis se propone un modelo de precios hedónicos donde la variable dependiente es el precio de tasación de un conjunto de viviendas localizadas en la Región Metropolitana de Barcelona $(\mathrm{RMB})$ y las independientes son los diversos factores que inciden en la formación espacial de dicho valor.

El estudio, entre otras cosas, permite detectar, a escala metropolitana, cuales son los factores locacionales que se valoran positiva y negativamente. Esta información objetiva es importante para tomar decisiones sobre proyectos de inversión en vivienda de protección oficial y libre, para comprender las decisiones de ubicación y crecimiento metropolitano, para facilitar a las entidades financieras, aseguradoras y consumidores potenciales comprender las diferencias en las tasas de apreciación entre las diferentes zonas metropolitanas minimizando riesgos e incumplimientos, asimismo, permite a los analistas de políticas públicas delimitar áreas de inversión inmobiliaria, determinar un indicador para el control de impuestos urbanos (predial, catastro y valorización).

La investigación define nuevas líneas de evaluación urbana a una escala metropolitana, donde el resultado se aproxima a una valoración económica de la ciudad, permitiendo analizar la incidencia de atributos urbanos y naturales que le dan atractivo.

\footnotetext{
${ }^{1}$ Autor de la Tesis. Profesor Titular de la Facultad de Arquitectura de la UANL, México. jitch@far.uanl

${ }^{2}$ Director de la Tesis. Profesora y Directora adjunta del Centro de Política de Suelo y Valoraciones de la Universidad Politécnica de Cataluña. pilar.garcia-almirall@upc.edu

${ }^{3}$ El artículo es producto de la tesis doctoral titulada con el mismo nombre, leída el 15 de Marzo del 2006 en la Escuela Técnica Superior de Arquitectura de Barcelona de la Universidad Politécnica de Cataluña. FITCH Osuna, Jesús Manuel (2005): La incidencia de externalidades ambientales en la formación espacial de valores: El caso de la Región Metropolitana de Barcelona. Tesis Doctoral. Universidad Politécnica de Cataluña.
} 


\section{Introducción}

La relación del hombre con la naturaleza, ha existido desde el origen mismo de la vida humana. Dicha relación, ha conllevado a materializar los procesos naturales en lo que denomina un medio ambiente construido o artificial (ciudades), que se superpone al medio ambiente natural. Lo que se denomina "ambiente urbano", considerado como una cuestión central de política económica y social en el modelo de ciudad sustentable discutido recientemente en la OCDE. Sin embargo, las áreas urbanas se han visto como concentraciones en las que se sufre una degradación ambiental, con elevada contaminación atmosférica, ruido y congestionamiento vial que afecta la salud y el bienestar de sus habitantes. En este sentido, la sustentabilidad urbana es, según Nijkamp y Perrels (1994), alcanzar cualitativamente un nivel socioeconómico, demográfico y tecnológico que permita mantener el funcionamiento de la ciudad en el largo plazo. El estudio de las externalidades y de su impacto sobre el sistema urbano es un elemento clave de cara al diseño de ciudades sostenibles. De manera que el estudio de las externalidades es el tema central de análisis de la economía ambiental iniciada a partir de las aportaciones de Coase (1960). La cuantificación económica de las externalidades ha estado basada generalmente en resultados experimentales obtenidos por métodos estadísticos (Constanza, 1991; Hartwick, 1977; Tietemberg, 1988; Daly, 1989; Johansson, 1990; Hausman, 1993; Pearce y Turner, 1990; Pearce 1993; Cummings y Harrison 1995, entre otros). En la década de los 90's se desarrollaron numerosas aplicaciones en España a partir de Naredo (1993), entre otras, cabe destacar las obras de Azqueta (1994), Azqueta y (1996), Campos y Riera (1996), Riera (1994 y 1995) y Riera et al., (1994).

Siguiendo esta línea, esta investigación contribuye con instrumentos que evalúan la realidad urbana de una manera objetiva. Considerando efectos negativos (la contaminación atmosférica, ruido, degradación y obsolescencia del patrimonio urbano edificado...) y positivos proximidad a sitios de interés, accesibilidad, diversidad y cualificación de usos de suelo, equipamientos y servicios, características de la población, calidad del trasporte público...) entre otros factores que inciden en la formación y estructuración de los valores urbanos. La valoración económica urbana nos ayuda, entre otras cosas, a discernir el nivel de preferencia asignado por un consumidor a un bien inmueble. El objetivo del estudio es describir los atributos que son valorados al momento de la elección residencial. Para ello se emplea la base de información de la empresa Colectivo Arquitectos Tasadores, Sociedad Anónima (CATSA). A partir de una muestra de 24,340 tasaciones de vivienda realizadas en la Región Metropolitana de Barcelona (RMB) durante el periodo del 2001 al 2002, se describe la formación espacial de valores (valores medios por municipio) y específicamente la incidencia de las externalidades ambientales en este fenómeno. La metodología utilizada en la investigación es la técnica Multivariante de los Precios Hedónicos o regresión hedónica.

\section{Externalidad ambiental y valor inmobiliario}

El carácter inmóvil de los bienes inmuebles hace que en el momento de su adquisición, se obtengan conjuntamente una serie de atributos que complementan el objeto de uso del bien: características espaciales, (como la localización respecto a otras unidades residenciales, al empleo y a otras instalaciones) características del barrio, estrato social y nivel de educación de los residentes, entre otros. Esto implica que puedan existir importantes externalidades inherentes a la localización elegida.

En el caso relacionado a la vivienda, las externalidades se han clasificado principalmente en tres bloques: externalidades físicas, sociales y desertización urbana (López García, 1992ª González-Páramo y Onrubia, 1992). 
Las externalidades físicas están referidas a los valores positivos o negativos que afectan al entorno del inmueble. Los aspectos relacionados a la contaminación atmosférica y sonora son, sin duda, algunos de los más estudiados en la literatura como efectos negativos de las externalidades. Otro efecto negativo es la falta de regulaciones en el ámbito colectivo sobre riesgos de incendio o problemas de tipo sanitario para la vecindad; así como la ausencia de cierta limitación sobre los usos posibles de suelo puede crear externalidades espaciales, del tipo de construcción de viviendas próximas a vías de comunicación. En el sentido contrario encontramos también externalidades positivas, como por ejemplo, la cercanía a una zona verde. En definitiva, el efecto de las externalidades sobre los precios inmobiliarios se basa en el bienestar o malestar que no se negocia en el mercado. Por ejemplo en el caso de las zonas verdes no se incurre en gasto alguno para gozar de su belleza. Sin embargo, produce un aumento del bienestar de los individuos. En este sentido, las externalidades se internalizan en el valor de los bienes privados urbanos produciendo un efecto de precios sombra.

La rehabilitación de una fachada, por ejemplo, aumenta la utilidad de los paseantes y de los que viven en el barrio. Estos efectos externos, llamados efectos vecindad, hace que se genere una cierta apreciación, lo cual repercute en un aumento del precio de la vivienda. En este sentido vemos que la ciudadanía forma parte esencial en el quehacer de nuestras ciudades, lo cual mediante su participación se le puede dar aumento de valor a nuestro patrimonio.

Las externalidades sociales se definirían como aquellos valores positivos o negativos atribuibles a las características demográficas de una determinada zona. La segregación residencial, segmenta la ciudad de acuerdo a la clase social, etnia o religión formándose los conocidos ghettos o barrios marginales, donde se concentran familias de bajo poder adquisitivo.

La desertización demográfica es otra externalidad, producida en la mayoría de los barrios céntricos de las ciudades. La duración de vida útil de las viviendas, produce con el tiempo obsolescencia funcional tanto en el espacio público como en el privado. Lo que incita un proceso de migración hacia otros sitios con mayor calidad ambiental y del parque edificado. En el sector desertizado se inician efectos vecindad y un bajo nivel de vida de sus ocupantes, haciendo que se originen zonas con viviendas deterioradas e infrautilizadas. Los inmuebles tienden a deteriorarse y ser abandonados ya que el sistema de mercado no permite su rehabilitación. Es decir el precio o renta no es suficiente para cubrir los costes de operación necesarios en la rehabilitación. Estas condiciones sociales explican, en ocasiones, las decisiones de invertir en viviendas de nueva construcción, en determinados barrios, mientras viviendas que reúnen buenas condiciones de calidad y precio son infrautilizadas en otras barriadas, pese a suponer un ahorro monetario (Jaén y Molina; 1993).

\section{Modelos teóricos que incorporan las externalidades ambientales}

Alfred Marshall a finales del siglo XIX, orientó sus estudios hacia el conflicto que se origina entre los intereses privados y públicos fruto del libre mercado. El equilibrio del mercado se consigue con unos límites de intensidad de usos (en intensidad de edificación y en ocupación de suelo) que pugnan con el óptimo social. No obstante, no es solamente el óptimo social, entendido éste como productividad, lo que se resiente de dicho funcionamiento del mercado, sino incluso la misma propiedad privada. De ahí que la imposición de un «impuesto de aire puro no sería una carga muy pesada para los propietarios, puesto que buena parte del mismo les sería retornado bajo la forma de un aumento de valor de los terrenos restantes».

Los supuestos básicos del análisis económico tradicional neoclásico son los de competencia perfecta en el mercado y divisibilidad total de recursos, bienes y servicios. En este contexto se postula la capacidad del mercado para lograr automáticamente un óptimo económico en términos de bienestar social u óptimo de Pareto. En este óptimo toda influencia económica de 
la conducta de una persona (o de una firma) sobre el bienestar (o los beneficios o costos) de otra persona (u otra firma) se trasmite a través del mercado, y concretamente mediante la modificación de los precios. Siguiendo este planteamiento, la teoría tradicional de la demanda, sugiere que los individuos, actuando racionalmente, tienden a maximizar su utilidad en relación a un presupuesto limitado por sus ingresos. Lo cual se expresa mediante la siguiente función:

$$
U=\left(X_{1}, X_{2}, \ldots, X_{n}\right)=U\left(X_{i}\right)
$$

Donde $U$ corresponde al nivel de utilidad y $X_{i}$ la cantidad de la comodidad $i(i=1, \ldots, n)$ consumida por el individuo. La limitación del presupuesto vendría dado por:

$$
Y=\sum_{1=1}^{h} P_{1} X_{1}
$$

Yamada (1972), mediante esta concepción plantea la siguiente función de utilidad:

$$
u=u\left[c, s, o^{e}, o^{w}, o^{c}(r), q^{e}(r)\right][3]
$$

Donde $c$ son todos los demás bienes, $s$ la cantidad de espacio consumido, $O^{e}$ el tiempo utilizado en el ocio, $O^{W}$ el tiempo de trabajo, $O^{C}(r)$ el tiempo de viaje al trabajo, $q^{e}(r)$ la calidad ambiental y $r$ la distancia al DNC. Extiende la consideración del modelo estándar ${ }^{4}$, involucrando al ocio y la calidad ambiental como elementos determinantes en la formación espacial de valores. Este supuesto corresponde al funcionamiento de una ciudad monocéntrica típica, con una función decreciente de la renta con la distancia y una función creciente de la calidad ambiental con el incremento de accesibilidad, al estar fuertemente interrelacionada la primera con la densidad y decrecer ésta con la distancia al centro de negocios. Siguiendo a Richardson, el autor señala que el modelo es limitado al venir determinados exógenamente los costos de transporte, la densidad (responde inversamente respecto a la calidad ambiental) y, sobre todo, la renta del suelo. El hecho, en particular, de que la función de esta última se considere necesariamente negativa (decreciente) en relación a la distancia, resta buena parte de las posibilidades de ese modelo para explicar los casos en los cuales existen gradientes de renta positivos.

El trabajo de Papageorgiou (1976), también hace aportaciones relevantes en este sentido. A diferencia del modelo de Yamada, su modelo permite también implementarse en un tratamiento policéntrico y jerarquizado. Introduce el tiempo obtenible por el consumo (o por el ocio) y la calidad ambiental como variables dentro de la función de utilidad del consumidor:

$$
U=u[c, s, t(r), e(r)]
$$

Donde $c$ son todos los demás bienes, el espacio consumido, $t(r)$ el tiempo obtenible por el consumo y $e(r)$ las amenidades residenciales del entorno. En el caso de sistemas urbanos policéntricos interesa destacar que la inclusión de la variable $e(r)$ (responde proporcionalmente respecto a la calidad ambiental) permite la aparición de gradientes de renta positivos e incluso irregulares. Otro resultado es la modificación de la estructura de coronas concéntricas del modelo estándar, adquiriendo una forma discontinua, incluso en el marco de un espacio continuo.

Richardson (1977) inducido por la relación que presentan las amenidades de los barrios y la calidad ambiental en los valores del suelo, plantea un modelo en el que considera que ciertos lugares específicos otorgan ventajas adicionales a la accesibilidad al centro de negocios.

\footnotetext{
${ }^{4}$ El modelo estándar se refiere a la concepción de la formación de los precios del suelo en el intercambio, o trade off, entre accesibilidad (centro de laciudad) y valor.
} 
Dichas ventajas pueden aparecer por gran cantidad de influencias, pero en los modelos de localización residencial, las más comunes son las externalidades del barrio. Por su parte, la densidad, incorporada en la función de utilidad sugiere que los usuarios desean más habitar en densidades bajas que en elevadas. Para ello considera dos casos. En el primero expresa la función de utilidad planteando que todos los usuarios tienen los mismos ingresos:

$$
U=u(c, s, \breve{s})
$$

Donde $c$ son todos los demás bienes, $s$ la cantidad de espacio consumido y š un indicador del tamaño de la parcela por vivienda en el barrio. En el segundo caso considera la posibilidad de familias de rentas desiguales:

$$
U=U\left(c, s, \check{s}, O^{e}, O^{c}, O^{w}\right)
$$

Donde $c, s$, š tienen la significación anterior, $O^{e}$ es el tiempo de ocio, $O^{c}$ el tiempo de viajes y $O^{W}$ el tiempo de trabajo. Mediante este planteamiento se generaliza y teoriza el concepto de renta de externalidad urbanística que es utilizado en trabajos que pretenden evaluar componentes adicionales a la accesibilidad en la formación espacial de valores.

La incidencia de las características físicas se considera como un elemento determinante de los valores, calculándose a través de la regresión múltiple sus precios al actuar como variables independientes. En esta línea se han abordado dos tipos de externalidades: ambientales y urbanísticas. Ambas se han conjugado, con el objetivo de mejorar la interpretación de los valores, incluyendo en sus análisis aspectos como la congestión del tráfico, el ruido, la tranquilidad ciudadana, la cantidad y calidad de los equipamientos públicos y de los espacios libres, la densidad, el deterioro del parque edificado, los impuestos y la zonificación, entre otros.

La argumentación de estos estudios se basa en que las externalidades tienden a tener una plena materialización físico/urbana, pudiéndose considerar como diversas características del espacio y calcularse su incidencia en los precios por medio del análisis de regresión, determinando sus precios implícitos.

$$
P=a_{1} X_{1}+\ldots+a_{n} X_{n}+e[7]
$$

Donde $X_{1}, i=1, \ldots, n$, son las cantidades de las $i$ características determinantes de los precios, y $a_{1}$ el precio implícito de cada una de aquéllas. El precio implícito es, pues, la contribución marginal de una variable, constantes las demás, en el precio, y puede ser definido como $\partial \mathrm{P} / \partial \mathrm{X}_{1}$.

\section{El Método de los Precios Hedónicos (MPH)}

Los trabajos que se inician en la teoría de la externalidades se fundamentan con conceptos de la teoría de la demanda, mediante lo cual se realizan estudios empíricos en la comprensión de la formación de los precios del suelo (y de la vivienda) en diversas áreas urbanas. Grilliches (1971) y Rosen (1974) son considerados, en la literatura especializada, los padres de la técnica del análisis de precios hedónicos. El método consiste en desglosar el precio de un bien privado, en función de varias características. Estas características tienen un precio implícito cuya suma determina, en una proporción estimable, el precio del bien de mercado que se observa. Así, el precio de una vivienda puede determinarse por la agregación de los precios implícitos de sus características y de las del entorno en el que está ubicada. Mediante procedimientos econométricos se calcula el peso de las variables que determinan el precio final de la vivienda (por ejemplo, superficie de la casa y de la parcela, tipología, número de habitaciones, de baños, antigüedad, distancia al centro de la ciudad, nivel de polución 
atmosférica o atractivo del paisaje) y, bajo determinados supuestos, se estiman los precios de dichas características.

El valor del bien inmobiliario expresa los beneficios que se pueden tener del mismo, a través del alojamiento, pero hay otros como el acceso al lugar del trabajo, a las instalaciones comerciales y atributos ambientales, como la calidad ambiental del vecindario en el que está ubicado el terreno también son beneficios importantes que recibe la persona que tiene el derecho al usar un determinado inmueble. Las distintas ubicaciones tienen distintas atribuciones ambientales, tales valoraciones darán lugar a diferencias en los valores de la propiedad.

El método hedónico emplea la estadística inferencial para diferenciar los atributos de dichas ubicaciones, basándose en dos puntos principales:

- Identificar la cantidad diferencial del valor de las propiedades que se debe a las diferencias ambientales entre las mismas.

- Inferir cuántas personas estarían dispuestas a pagar por una mejora de la calidad ambiental con la que se encuentran y cuál es el valor social de la mejora.

La identificación de las diferencias entre las viviendas conlleva a establecer una serie de variables, las cuales pueden ser: de la propiedad, de vecindario, de accesibilidad y ambientales. Si se excluye una variable que este muy correlacionada los efectos estimados sobre el valor de la propiedad pueden tener sesgos, ya sea hacia arriba o hacia abajo. Lo cual dependerá de cómo se encuentran relacionados entre sí las variables incluidas y excluidas y el valor de la propiedad. Por otro lado, si el estudio incluye una variable irrelevante no se produce un sesgo tan sistemático, aunque las estimaciones de los efectos de las variables incluidas sean menos fiables.

El primer paso en el método de precios hedónicos es la estimación de una ecuación del estilo:

Precio del bien $=f$ (variables de propiedad, de vecindario, de accesibilidad, ambientales)

Lo cual se puede expresar matemáticamente con la siguiente fórmula:

$$
x=a_{0}+a_{1} v_{1}+a_{2} v_{2}+\ldots+a_{n} v_{n}
$$

Donde:

$X \quad=$ precio del inmueble por $\mathrm{m}^{2}$.

$V_{1} \quad=$ variable exógena o signo externo número 1 (p.ej.: Accesibilidad).

$V_{2} \quad=$ variable exógena o signo externo número 2 (p.ej.: calidad ambiental).

$V_{n} \quad=$ variable exógena o signo externo número $n$ (p.ej.: características sociales $\mathrm{y}$ económicas de la población).

$a_{o} \quad=$ término independiente o constante de la ecuación.

$a_{1}, a_{2}, \ldots, a_{n} \quad=$ coeficientes de las variables exógenas.

Los procedimientos indirectos calculan una relación "dosis-respuesta" entre la contaminación y algún efecto y sólo entonces hay alguna medida de la preferencia para el efecto aplicado. 
Ejemplo de ello tenemos: el efecto de la contaminación sobre la salud, la depreciación física de activos materiales (metales y construcciones), ecosistemas acuáticos y sobre la vegetación. En el caso de nuestra investigación afecta en la valoración económica de los inmuebles, producida por la perdida el bienestar de las personas. ${ }^{5}$

Las principales críticas de este método no difieren de las que en general pueden hacérsele a otros de similar naturaleza, p.e.: inadecuada especificación o desconocimiento de la verdadera forma funcional, carencia y/o inadecuada información de los atributos de la propiedad, omisión de variables relevantes, frecuentes problemas de multicolinealidad, etc. A pesar de estas posibles limitaciones, los precios hedónicos son cada vez más utilizados por organismos estadísticos oficiales de diversos países para calcular índices de precios al consumidor, en especial porque permiten controlar eficazmente la heterogeneidad de los productos y sus rápidos cambios cualitativos a través del tiempo. Roca (1988), hace mención de dos cuestiones referentes a la utilización de la técnica. La primera señala que para que funcione el análisis de regresión para cuantificar de forma fiable los precios implícitos, las variables o características configuradoras de los precios han de ser tendencialmente separables entre sí, refiriéndose con ello al fenómeno de la multicolinearidad, donde puede implicar la pérdida formal de la significación estadística de las variables. Recomienda para ello manipular anteriormente las variables presuntamente explicativas de los precios, a través de las técnicas de análisis de componentes principales y/o el análisis factorial. Con lo que se puede llegar a unos factores explicativos de los precios, totalmente independientes (ortogonales) entre sí. La segunda cuestión es que la forma lineal que aparece en la ecuación [8] no es absolutamente necesaria, pudiéndose llegar a ecuaciones mucho más complejas (logarítmicas, semilogarítmicas, etc.), que eventualmente pueden explicar mejor la contribución de las diversas variables en la formación de los precios.

A partir de Grilliches (1971) y Rosen (1974) se han elaborado numerosos estudios que pretenden medir el significado de los precios implícitos bajo determinadas condiciones, identificándose la función de demanda de la característica escogida y, en consecuencia, el excedente del consumidor. Así, variaciones en la provisión de tal característica (por ejemplo, nivel de polución) comportan diferencias, medidas en unidades monetarias, en el bienestar de las personas.

Manning (1986), sobre la base de los trabajos de Rosen (1974), Roback (1982) y Graves (1980), ha analizado los factores que explican las diferencias en el precio de la viviendas entre ciudades e introduce un concepto interurbano de equilibrio en la calidad de vida residencial (interurban household QOL equilibrium). Su principal aportación sugiere que una parte de la población está dispuesta a renunciar a cierta parte de su renta potencial por tal de vivir en ciudades con amenidades extraordinariamente atractivas. Es decir, a ver incrementada su utilidad no por la remuneración a su trabajo, sino por vía de las externalidades. Por su parte el modelo de equilibrio de Krumm (1960) constata que, tras haber considerado los atributos de la vivienda, los factores explicativos del precio son la homogeneidad del barrio y el nivel de servicios. El modelo hedónico propuesto por Palmquist (1984), calibrado para 7 Standard Metropolitan Statistical Areas (SMSA) estadounidenses ${ }^{4}$, tiene la peculiaridad de derivar las correspondientes curvas de demanda. En su estudio plantea dos hipótesis. En la primera considera que el mercado de cada ciudad no está segmentado, y en la segunda que los elevados costes impedían la movilidad perfecta entre ciudades. Los resultados obtenidos señalan, que la condición racial tiene una gran influencia negativa en el precio de la vivienda,

\footnotetext{
${ }^{5}$ Sobre la tasación de apartamentos en barrios de Madrid (Argüelles-Gaztambide), utilizando el análisis de regresión, así como el análisis factorial, las clasificaciones cluster y otras técnicas estadísticas, véase LOZANO, J. (1996), Tasación urbana: Una metodología para informes de tasación masiva, Tesis doctoral, Universidad Politécnica de Madrid. Véase también KINCHELOE, S.C. (1993). "Linear Regression Analysis of Economic Variables in the Sales Comparison and Income Approaches". The Appraisal Journal, LXI, no4, pp.576-586.

${ }^{4}$ Atlanta, Denver, Houston, Louisville, Miami, Oklahoma y Seattle
} 
al igual que el porcentaje de población transeúnte. Desde la perspectiva contraria, es decir, la de los factores que inciden positivamente en el valor, encuentra que las variables relacionadas con la educación de los vecinos y la ausencia de viviendas hacinadas son factores relevantes. Respecto a la incidencia la calidad del aire sobre el precio de las viviendas, sólo en 4 de las 7 SMSA estudiadas este factor tuvo significancia estadística. Este resultado obedece a que Palquimist su intención no era la de valorar la calidad del aire, más bien estimar una función de demanda de las distintas características de la vivienda. Roback (1989) estudia las amenidades locales en la decisión de ocupación y desarrolla un ranquin entre las áreas metropolitanas según los factores QOL.

Blomquist, Berger i Hoehn (1988) estudian los factores QOL, en 253 provincias y pone de manifiesto que el mercado inmobiliario integra en sus valores diferenciales una estrecha proporcionalidad con la variación de dichos factores. Así, los modelos hedónicos elaborados permiten medir acusadamente la calidad de las viviendas y el nivel de servicios asociados. El resultado en base a análisis multidimensionales sobre aspectos QOL tiende a fijar el ambiente físico y las oportunidades culturales y recreativas con una mayor significancia de la calidad urbana.

En esta línea, García Almirall (1997) establece medidas indicativas del valor implícito de ciertas variables medioambientales, que vienen a internarse en los valores urbanos, utilizando el análisis estadístico multidimensional y técnicas de análisis espacial SIG. Su aportación, constituye un primer paso clave en el desarrollo de modelos explicativos de la diferenciación y estructuración de los valores urbanos con una mayor integración de variables medioambientales urbanas.

Clark (1997) plantea que los factores de calidad de vida son determinantes de una ciudad sustentable. Realiza un estudio mediante la técnica de los precios hedónicos, bajo la hipótesis de que además de los factores físicos, los relacionados con la etnia influyen sobre la determinación del valor inmobiliario. Para analizar este fenómeno agrupa las variables en 3 bloques (variables de amenidades y desamenidades ambientales, variables fiscales y variables nocivas) correlacionado a los precios implícitos de vivienda de tres categoría de personas (blancos no hispánicos, negros e hispanos). Este tipo de estudios contribuye que los efectos sociales son parte de las externalidades ambientales que se materializan en el territorio.

\section{El Caso de la Región Metropolitana de Barcelona}

Como se ha dicho antes, el ámbito de estudio, corresponde al ámbito metropolitano de Barcelona (RMB), conformado por 7 comarcas: El Barcelonés, El Baix Llobregat, El Garraf, El Maresme, El Vallès Occidental y El Vallès, Oriental. Dichas comarcas tienen un total de 164 municipios y una población agregada de 4,390,390 habitantes. La media es de 26.670 habitantes por municipio. El municipio que tiene más población es Barcelona, con 1.503.884 habitantes, y el de menor número es Granera.

En este sentido el análisis de los datos se realizará mediante la técnica de regresión múltiple y el análisis factorial, con lo que se podrá obtener un modelo de precios hedónicos. Posteriormente los resultados se contrastaran con mapas temáticos producidos por los Sistemas de Información Geográfica (SIG). Con ello se generará un buen nivel de análisis del ámbito de estudio y sobre todo la comprobación de la hipótesis planteada. La metodología a emplear se basa en la literatura producida a partir de los trabajos de Rosen (1974): Clark (1997); Palmqist (1984). Y modelos globales que introducen variables de accesibilidad, calidad urbanística y jerarquización social: Roca (1986); García (1997 y 2000). Así como modelos econométricos a nivel nacional, autonómico y provincial: Caballer et. al. (2002). Mientras que a 
través el trabajo de Narváez (2002) sobre las relaciones sociales-físicas que se sucintan en el espacio será utilizado como referente en este aspecto.

\section{Bases de información}

El Sistema de Información Geográfica (SIG) integra la información de las siguientes fuentes:

- Centro de Políticas de Suelo y Valoraciones (CPSV)

- Colectivo Arquitectos Tasadores Sociedad Anónima (CATSA)

- Fundación "La Caixa"

- Anuario económico

- Anuario social

- Instituto Nacional de Estadística (INE)

- Instituto de Estadística de Cataluña (IDESCAT)

- Generalatitat de Cataluña

- Servicio Meteorológico de Cataluña

- El aire

- Agencia de Residuos de Cataluña

- Centro de Investigación Ecológico y Aplicaciones Forestales (CREAF, en sus siglas catalanas)

- Ministerio de Fomento

- Catastro Estatal

- MCRIT

\subsection{Descripción de la base de información de valores inmobiliarios (CATSA)}

Los valores inmobiliarios provienen de las tasaciones de CATSA, la cual ha realizado 39.425 valoraciones durante el 2001 y el 2002. En la tabla 1, se observa la distribución por ámbito destacando la proporción de tasaciones en la Comunidad Autónoma de Cataluña.

En relación con los datos de Ministerio de Fomento, para el ámbito de Cataluña y durante el periodo anteriormente señalado se tienen 236.635 tasaciones, lo que representa proporcionalmente un $13,75 \%$ de las valoraciones de CATSA. Para la RMB hay 155.077 tasaciones y su proporción $15.70 \%$, mientras que el municipio de Barcelona tiene 38.520 tasaciones que representa un $15,00 \%$.

Tabla 1. Número de tasaciones por ámbito

$\begin{array}{ccc}\text { Ámbito } & \begin{array}{c}\text { No. } \\ \text { Tasaciones }\end{array} & \begin{array}{c}\text { \% respecto a la } \\ \text { muestra }\end{array} \\ \text { España } & 39.425 & 100 \\ \text { Cataluña } & 32.536 & 82,53 \\ \text { Región Metropolitana de Barcelona } & 24.340 & 61,74 \\ \text { Barcelona } & 5.773 & 14,64\end{array}$




\section{Mapa 1. Localización geográfica de las tasaciones de CATSA}

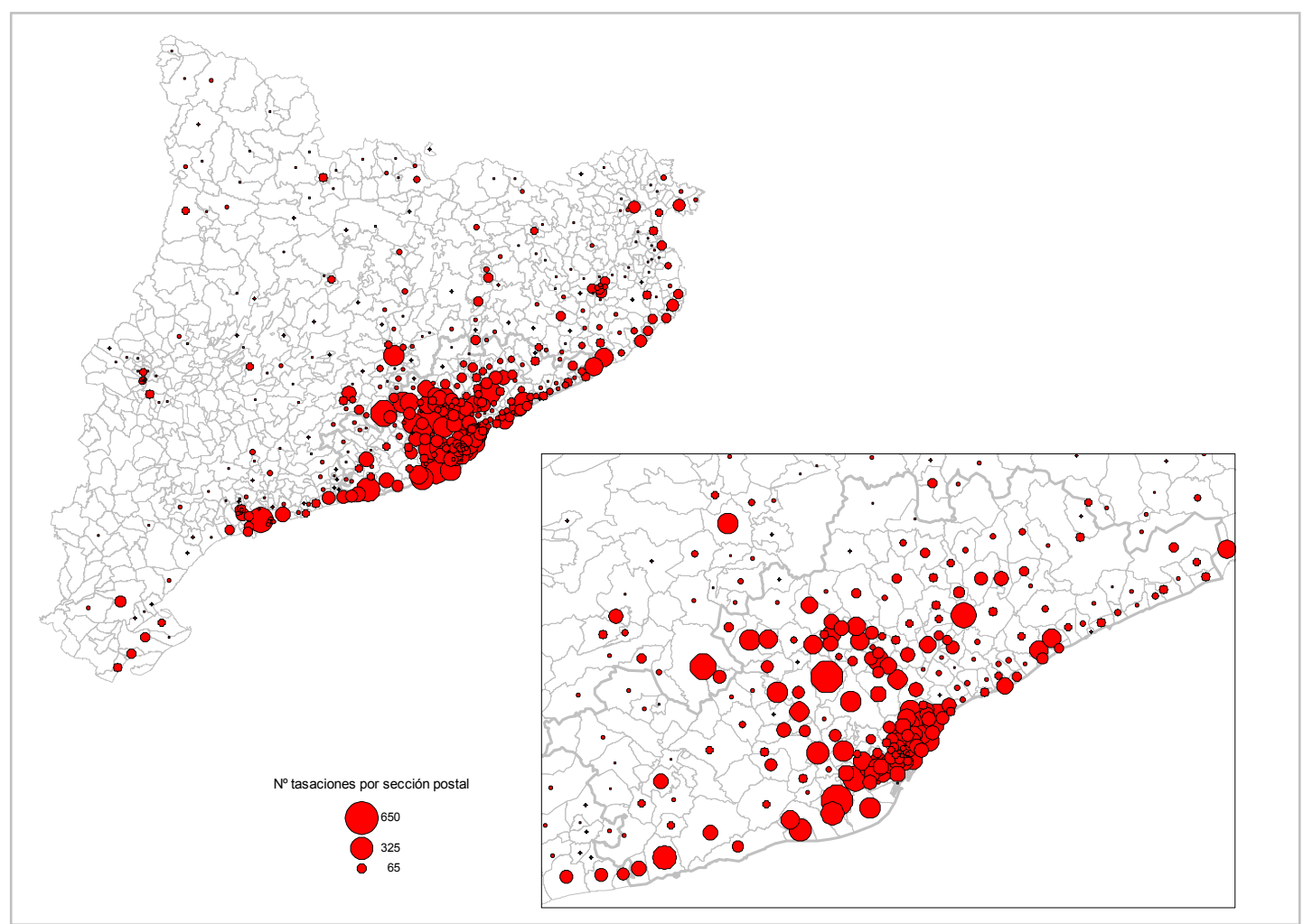

Fuente: elaboración propia

\section{Análisis descriptivo de los precios}

El análisis descriptivo de los precios se ha realizado a través de los estadísticos: desviación estándar, máximo, mínimo y media. Considerando los ámbitos espaciales de Cataluña, Región Metropolitana de Barcelona y la Región Metropolitana excluyendo a Barcelona. Para el caso de Barcelona se señala el valor correspondiente del indicador. Posteriormente, se realiza un análisis de correlación en función de la captura de información en la elaboración de las tasaciones. Esto queda implícito en tres dimensiones, a partir de las cuales se estudian los inmuebles:

- Dimensión accesibilidad

- Dimensión socioeconómica

- Dimensión urbana-ambiental 
Tabla 2. Variables positivamente correlacionadas con el precio: RMB

Variable MF2001 MF2002 CATSA

Linealización acabados pavimento estar

0,69

0,70

Componente Principal 1: Nivel instrucción

0,52

0,58

0,61

clasificación de municipios todas las variables

0,55

0,58

0,60

clasificación de municipios_eco

$0,58 \quad 0,58$

0,60

clasificación de municipios_variables vivienda

0,55

0,56

0,59

Gas inmueble

$0,64 \quad 0,62$

0,58

\%TP_SOP

0,56

0,58

0,57

GAS

0,63

0,61

0,56

Consolidación: $75 \%$

0,55

0,53

0,55

Componente Principal 2: Superficie

0,62

0,62

0,55

SERV_\%

0,58

0,61

0,55

Gas localidad

0,58

0,55

0,54

IRPF_MIG

0,51

0,55

0,53

$\% B U P \_C O U$

0,43

0,50

0,52

\%TITULO_S

0,43

0,48

Componente Principal 1: Socio Profesional

$0,43 \quad 0,48$

0,50

Tabla 3. Variables correlacionadas negativamente con el precio en la RMB

\begin{tabular}{lrrr}
\hline Variable & MF2001 & MF2002 & CATSA \\
\hline 1 a 2 Plantas & $-0,54$ & $-0,54$ & $-0,40$ \\
\%OP_INMAQ & $-0,35$ & $-0,40$ & $-0,40$ \\
Clasif. admin: otros & $-0,33$ & $-0,32$ & $-0,43$ \\
Eq. lúdico: deficiente & $-0,33$ & $-0,36$ & $-0,43$ \\
Entorno: rural & $-0,29$ & $-0,32$ & $-0,45$ \\
Llegada a la vivienda: 1941-1960 & $-0,44$ & $-0,42$ & $-0,49$ \\
Componente Principal 1: Parque vehicular & $-0,54$ & $-0,49$ & $-0,49$ \\
Eq. escolar: deficiente & $-0,45$ & $-0,44$ & $-0,50$ \\
\%C_INDCON & $-0,47$ & $-0,52$ & $-0,51$ \\
Superficie: 91 a 120 m2 & $-0,65$ & $-0,64$ & $-0,54$ \\
\%C_AG_PES & $-0,50$ & $-0,48$ & $-0,54$ \\
ACCRES & $-0,60$ & $-0,59$ & $-0,56$ \\
ACCTRE & $-0,60$ & $-0,59$ & $-0,56$ \\
Consolidación: 50\% & $-0,49$ & $-0,43$ & $-0,57$ \\
En propiedad: por herencia o donación & $-0,57$ & $-0,56$ & $-0,60$ \\
Desarrollo: lento & $-0,61$ & $-0,61$ & $-0,60$ \\
Llegada a la vivienda: Antes 1941 & $-0,58$ & $-0,58$ & $-0,64$ \\
LNDISBCN & $-0,73$ & $-0,71$ & $-0,67$ \\
DISTBCN & $-0,72$ & $-0,71$ & $-0,68$ \\
\%EGB_1 & $-0,72$ & $-0,77$ & $-0,75$ \\
\hline
\end{tabular}




\section{Primera fase: emulación del valor inmobiliario sin incluir atributos arquitectónicos del bien residencial.}

La primera fase de elaboración de modelos consiste en trabajar con variables económicas, sociales-demográficas, accesibilidad y de externalidad urbana. En este primer paso no se han incluido variables e indicadores de los atributos arquitectónicos de las viviendas que ofrecen las tasaciones. El objetivo de dividir el trabajo en dos etapas consiste en establecer el valor añadido que reflejan los indicadores de CATSA, así mismo ver que relación existen entre los valores de dichas institución respecto a otras (Ministerio de Fomento) en la elaboración y formulación de modelos de valoración de vivienda.

El método utilizado en la determinación de los modelos económetricos es el de pasos sucesivos. El primer paso del método consiste en obtener un algoritmo de regresión, para posteriormente elegir las variables que mejor expliquen el fenómeno en cuestión. En relación con los otros métodos estadísticos, tiene la ventaja de admitir que una variable seleccionada en un paso puede ser eliminada en otro posterior.

El modelo resultante (Tabla 5) tiene 5 variables con un nivel de explicación del $87 \%$ de la varianza $\left(R^{2}=0,87\right)$, siendo su error estándar de $90,81 € / \mathrm{m}^{2}$. Las variables que explican la formación espacial de valores en la RMB son las siguientes:

- Factores de accesibilidad: DIST. (Distancia en Km al municipio de Barcelona)

- Factores Sociales: \%TITULO_S. Porcentaje de personas con titulo superior)

- Factores económicos: N_ECO02 (Nivel económico del municipio), IRPF_EMP (Impuesto sobre la renta de las personas físicas).

- Factores de externalidad urbana: <_1941 (llegada a la vivienda antes del año de 1941), VIV/PAR (Cociente del número de viviendas entre el número de parcelas).

La variable DIST, corresponde a la distancia en Km de los municipios de la RMB a Barcelona. Esta variable comprueba la teoría de las rentas de accesibilidad, sugerida seminalmente por von Thünen (1826) en su modelo de los cinturones agrícolas. Posteriormente Hurd (1903) y Haig (1926) ampliaron esta teoría generando la hipótesis que el funcionamiento del mercado urbano basa su localización de residencia en relación con la distancia al trabajo. Durante la década de los sesenta Alonso (1964), Wingo (1961) y Muth (1969) consideraron otros factores como el de uso de suelo, aportación que agrió una nueva línea teórica de análisis. Según Roca (1986), las formulaciones actuales de la teoría estándar tienden a identificar las decisiones de localización con un función de utilidad para cada consumidor, de manera que éste tiende a maximizar, además del conjunto de bienes no residenciales, la localización (entendida como accesibilidad al trabajo) y la cantidad de espacio consumido.

El segundo grupo está conformado por los aspectos sociales, estos factores se relacionan con la teoría de jerarquía social. En el modelo la variable que ha entrado corresponde al porcentaje de población con nivel de instrucción que tiene título superior, presentando una relación positiva según su coeficiente beta $(45,02)$.

Los indicadores económicos tienen considerable incidencia en la formación espacial de valores. En el modelo ingresan dos variables: el nivel económico y el IRPF ${ }^{7}$ de empresarios. La variable nivel económico ${ }^{8}$ calculada por la Caixa, presenta una relación positiva, lo cual es coherente con la realidad territorial, dado que el nivel de ingresos determina la adquisición de

\footnotetext{
${ }^{7}$ Impuesto sobre la Renta de las Personas Físicas.

8 Índice de la renta familiar disponible por habitante estimada por áreas geográficas (nivel municipal, provincial y por comunidades autónomas) para 2000.
} 
compra del producto inmobiliario. Este efecto lo complementa la variable que mide la renta de la población IRPF_EMP.

Los factores de externalidad urbana o amenidades urbanas se refieren al grupo de aspectos que hace deseable la localización espacial urbana, más allá de la accesibilidad y de los aspectos sociales. Las variables <_1941 y VIV/PAR forman parte de este grupo de variables. La variable <_1941, puntualiza la antigüedad media de los hogares en su vivienda actual es un indicador mūy interesante del dinamismo migratorio e inmobiliario de cada zona y aporta información muy útil sobre el proceso de formación de los hogares. En este caso el coeficiente beta da un signo negativo dado que en las zonas que se encuentra estos hogares presentan unas características obsoletas en cuanto a la utilidad arquitectónica, es decir las tecnologías van cambiando y dicho cambio se encuentra relacionado con funcionalidad de la vivienda. En algunos casos la falta de ascensor, la falta de mantenimiento, incide que los inmuebles forman parte de un segmento de viviendas para una clase social que no tiene los recursos económicos para migrar hacia lugares con mejores estándares de calidad arquitectónica.

La variable VIV/PAR, es el resultado de dividir el número de viviendas entre el número de parcelas a nivel municipal. El resultado es la densidad del municipio, a priori, aquellos municipios que tengan un mayor densidad tienden a tener una mayor concentración de actividad económica, y en esta medida, de servicios urbanos. Está variable puede servir para separar los municipios de carácter urbano de aquellos de tipo rural. El signo del coeficiente es positivo, indicando que los valores altos de vivienda se encuentran en los municipios más densos, es decir, en los más eficientes en términos de consumo de suelo, y con más servicios urbanos.

Tabla 4. Modelo explicativo de los valores de CATSA para la RMB

\begin{tabular}{|c|c|c|c|c|c|c|}
\hline Modelo & $\mathbf{R}$ & \multicolumn{2}{|c|}{$R$ cuadrado } & $\begin{array}{l}\text { R cuadrado } \\
\text { corregida }\end{array}$ & \multicolumn{2}{|l|}{$\begin{array}{l}\text { Error típ. de la } \\
\text { estimación }\end{array}$} \\
\hline 1 & ,933 & \multicolumn{2}{|c|}{ 870 } & ,863 & \multicolumn{2}{|l|}{90,81} \\
\hline & & \multirow{2}{*}{\multicolumn{2}{|c|}{$\begin{array}{l}\text { Coeficientes no } \\
\text { estandarizados }\end{array}$}} & e & RMB. & \\
\hline \multirow{2}{*}{\multicolumn{2}{|c|}{ Modelo }} & & & $\begin{array}{c}\text { Coeficientes } \\
\text { estandarizados }\end{array}$ & $t$ & Sig. \\
\hline & & B & Error típ. & Beta & & \\
\hline \multirow{7}{*}{$\begin{array}{l}(C \\
< \\
\% \\
V \\
N \\
\text { VIF } \\
D\end{array}$} & Constante) & 867,533 & 87,984 & & 9,860 & ,000 \\
\hline & 1941 & $-24,448$ & 2,827 &,- 471 & $-8,649$ &, 000 \\
\hline & İTITULO_S & 45,015 & 4,772 &, 507 & 9,433 &, 000 \\
\hline & /IV/PAR & 49,040 & 6,200 & 359 & 7,910 & 000 \\
\hline & J_ECO02 & 23,853 & 10,257 & 144 & 2,326 & ,022 \\
\hline & RPF_EMP & 15,177 & 4,229 & ,211 & 3,588 & ,000 \\
\hline & DIST & $-3,048$ & 898 &,- 187 & $-3,396$ & ,001 \\
\hline
\end{tabular}

a Variable dependiente: V_MEDIO 


\section{Segunda fase de emulación del valor inmobiliario incluyendo las características arquitectónicas de los inmuebles}

En la segunda fase se realiza un modelo en el que se incorpora variables arquitectónicas incluidas en las tasaciones de CATSA. A partir de lo cual se obtienen los resultados de la Tabla 6.

Tabla 6. Estadísticos descriptivos de las variables introducidas

\begin{tabular}{lrrr}
\hline Variable & Media & Desviación típ. & $\mathrm{N}$ \\
\hline V_MEDIO & 1262,52 & 248,37 & 143 \\
VIV_SEC\%_01 & 16,17 & 13,30 & 143 \\
IRPF_MIG & 2573,08 & 536,19 & 143 \\
LNDISBCN & 3,37 &, 536 & 143 \\
N. dormitorios & 3,37 &, 374 & 143 \\
Estado vivienda: proyecto & 2,10 & 4,77 & 143 \\
Consolidación: 75\% & 66,27 & 29,14 & 143 \\
Zonas verdes: abundante & 42,17 & 25,64 & 143 \\
Eq. comercial: abundante & 9,64 & 16,49 & 143 \\
Eq. lúdico: abundante & 5,89 & 10,18 & 143 \\
Calidad sanitarios: alta & 15,86 & 11,79 & 143 \\
Linealización Acabado pavimento estar & 1341,04 & 47,68 & 143 \\
Antes 1941 & 4,00 & 4,70 & 143 \\
\hline
\end{tabular}

El modelo presenta un nivel de varianza explicada del $91 \%\left(R^{2}=0,91\right)$ con 12 variables. La desviación estándar de los residuos es 76,43 , es decir un error de $76,43 € / \mathrm{m}^{2}$. Respecto al primer modelo presenta un aumento del $4 \%$ de explicación y el error ha disminuido en 14,38 $€ / \mathrm{m}^{2}$.

Tabla 7. Modelo de diferenciación urbana y de la edificación RMB

\begin{tabular}{ccccc}
\hline $\mathrm{R}$ & $\mathrm{R}$ cuadrado & $\begin{array}{c}\text { R cuadrado } \\
\text { corregida }\end{array}$ & $\begin{array}{c}\text { Error típ. de la } \\
\text { estimación }\end{array}$ & Durbin-Watson \\
\hline 0,96 & 0,91 & 0,91 & 76,43 & 1,96 \\
\hline
\end{tabular}

Las variables que explican la formación espacial de valores en la RMB son las siguientes:

- Factores de accesibilidad: LNDISBCN.

- Factores económicos: IRPF_MIG.

- Factores de externalidad urbana: Antes 1941, VIV_SEC\%_01, Eq. comercial: abundante, Zonas verdes: abundante

$\rightarrow$ Características vivienda: Linealizacion Acabado pavimento estar, N. Dormitorios, Calidad sanitarios: alta, Estado vivienda: proyecto, Consolidación: $75 \%$. 
Tabla 8. Coeficientes. Modelo de diferenciación urbana y de la edificación RMB

Modelo

Coeficientes no

Coeficientes

estandarizados

estandarizados

\begin{tabular}{lrrr} 
& \multicolumn{1}{c}{ B } & Error típ. & Beta \\
\hline (Constante) & 70,29 & 322,05 & \\
Linealización Acabado pavimento estar & 0,97 & 0,21 & 0,19 \\
Llegada vivienda. Antes 1941 & $-12,24$ & 1,81 & $-0,23$ \\
Eq. lúdico: abundante & 1,76 & 0,90 & 0,07 \\
IRPF_MIG & 0,16 & 0,02 & 0,34 \\
LNDISBCN & $-116,89$ & 19,69 & $-0,25$ \\
N. dormitorios & $-91,16$ & 23,60 & $-0,14$ \\
Estado vivienda: proyecto & $-5,12$ & 1,49 & $-0,10$ \\
Zonas verdes: abundante & 1,15 & 0,35 & 0,12 \\
Consolidación: 75\% & 1,28 & 0,36 & 0,15 \\
Calidad sanitarios: alta & 2,80 & 0,69 & 0,13 \\
Eq. comercial: abundante & 2,35 & 0,64 & 0,16 \\
VIV_SEC\%_01 & 2,39 & 0,67 & 0,13 \\
\hline
\end{tabular}

En el histograma de la Figura 1 se observa que la distribución de los residuos es aproximadamente normal. Si dicha distribución fuese perfectamente normal la media tendría que ser igual a cero y la desviación estándar cercana a uno. La desviación típica tiene un valor típico de 0,96 lo cual sugiere que no hay problemas en la validación de los supuestos en los que se basa la regresión MCO.

\section{Figura 1. Histograma del Modelo de diferenciación urbana y de la edificación RMB}

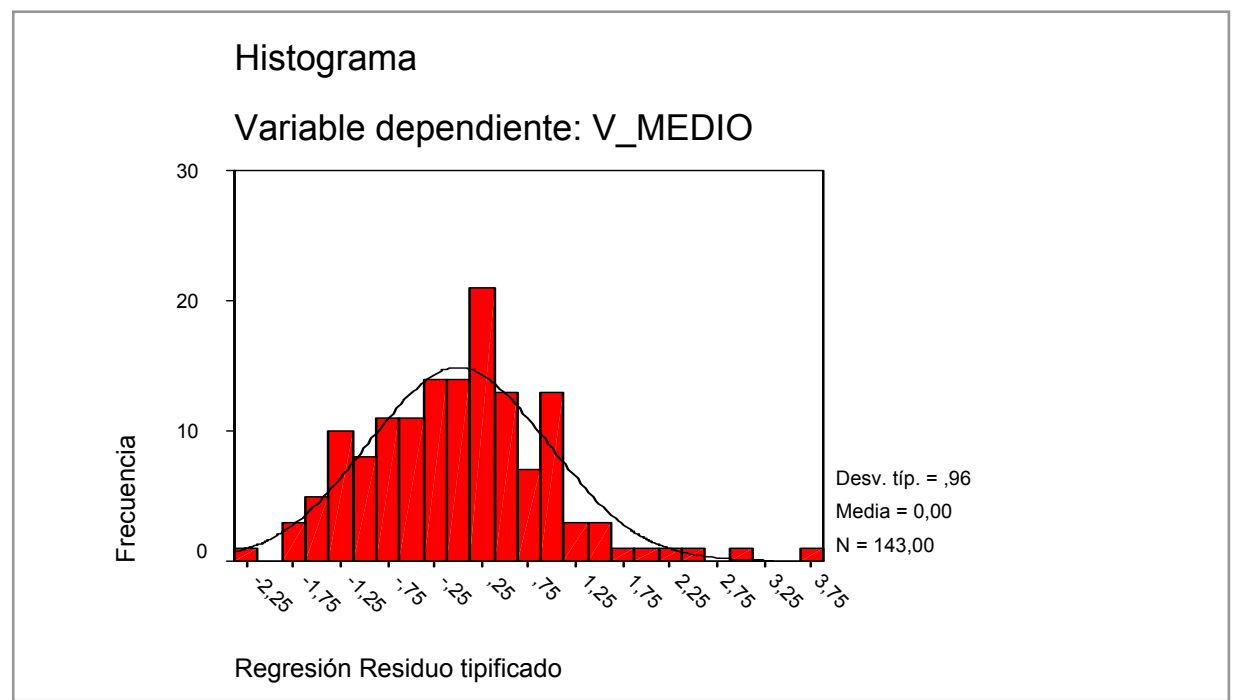

ACEO AÑO II, núm 6, febrero 2008 |LA INCIDENCIA DE LAS EXTERNALIDADES AMBIENTALES EN LA FORMACIÓN 
El gráfico P-P mejora la aproximación a la línea de referencia, respecto a los modelos anteriores.

Figura 2. P-P. Modelo de diferenciación urbana y de la edificación RMB

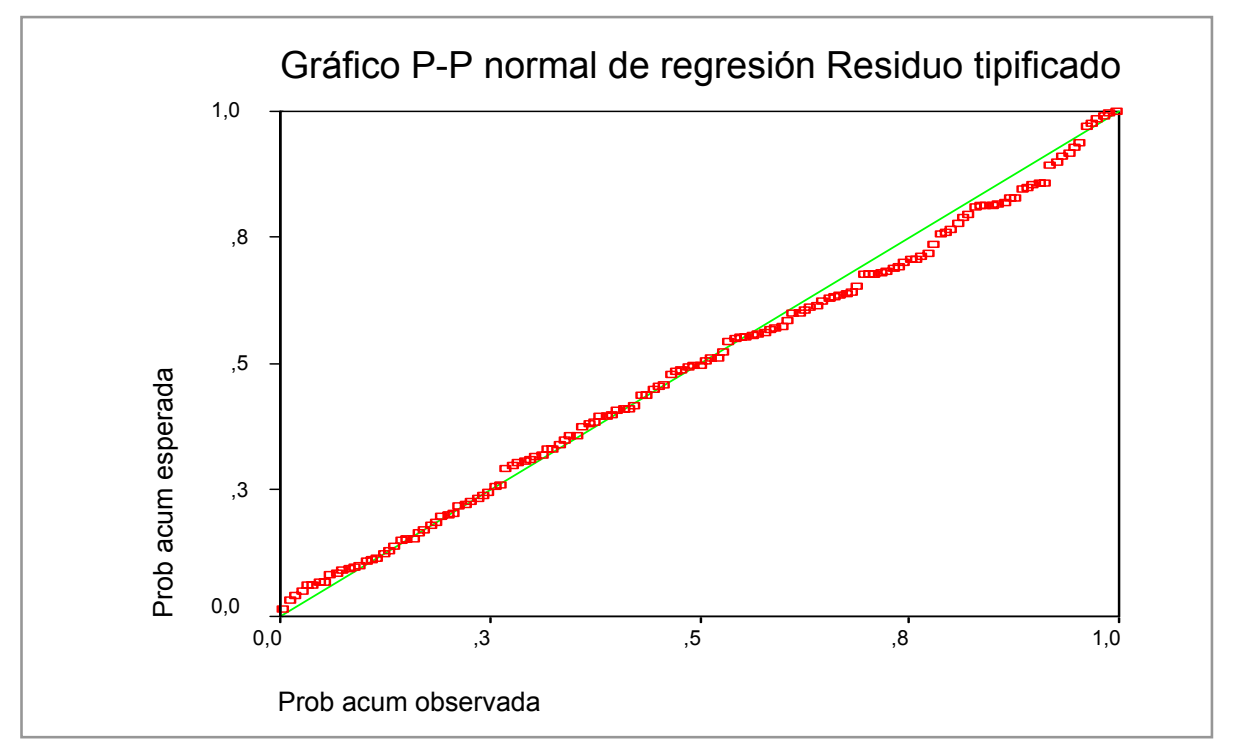

\section{Conclusiones}

La valoración ambiental de nuestras ciudades, entre otras cosas, permite entender la competitividad de las mismas, a través del impacto en términos de valor de los aspectos sociales, económicos, ambientales e institucionales que las caracterizan. La investigación aquí expuesta pretende aportar reflexiones teóricas y empíricas sobre la disposición a pagar por determinados bienes ambientales. En este caso se ha seleccionado un bien (vivienda), que al ser generalmente de tipo privado, no tiene los problemas de valoración de otros bienes ambientales no sujetos de titularidad privada; y por ende, sin mercado explícito. En este sentido se ha visto que el método de precios hedónicos es una herramienta analítica importante para inferir el valor de bienes que no tienen mercado como es el caso de los bienes y servicios ambientales. En este estudio hemos hecho uso de esta herramienta para estimar de manera indirecta el valor que los agentes económicos le asignan al entorno ambiental. La cercanía a la costa y a espacios con vistas espectaculares define una valoración positiva la cual repercute en las características de la vivienda, dimensión, el tipo de programa arquitectónico, acabados, entre otras variables que se encontraron en el estudio. Por otra parte se observa que los agentes no solo valoran variables ambientales de manera individual, sino que actúan con una racionalidad en la cual observan estas en su conjunto. En síntesis la tesis permite realizar sopesar la importancia de los servicios ambientales como factores de renta de localización en la Región Metropolitana de Barcelona a nivel de municipios. La escala municipal de análisis realizada en este trabajo deja una línea abierta en el sentido de realizar estimaciones a nivel de sección postal y de barrio para generar modelos que emulen espacialmente las principales determinantes del valor de la vivienda, así como otros tipos de inmuebles y submercados.

Sin embargo, desde la perspectiva estrictamente social, el problema pendiente de solución está relacionado con el acceso universal por parte de la población a las áreas de mayor 
calidad ambiental, en tanto éstas quedan reservadas, por medio del arbitrio de los precios inmobiliarios, a las personas con mayores ingresos. Esta problemática ya había sido señalada antes por Pearce (1993) quien constató que los más privilegiados, en el sentido de la solvencia económica, eran también los que consumían los mejores entornos en términos de calidad ambiental. McFadden y Leonard (1993) plantearon exactamente esa afirmación, en tanto Boercherding y Deaton (1972) y Bergstrom y Goodman (1973) encuentraron elasticidades ingreso por parques de recreación mayores que uno en la mayoría de los casos.

\section{Bibliografía}

ALONSO W. Location and Land Use. Harvard U.P., Cambridge, 1964

AZQUETA, D. Valoración económica de la calidad ambiental. McGraw-Hill: Madrid, España, 1994.

AZQUETA, D. y PÉREZ y Pérez, L. El valor económico de los servicios recreativos en los espacios naturales. Mc Graw Hill: Madrid, España, 1996.

BAILLY, A. L'organisation urbaine: théories et modèles. París, CRU. 1975. Traducción castellana: La organización urbana: teorías y modelos. Madrid. Instituto de estudios de administración local. 1978.

BERGSTROM, T.C. and GOODMAN R.P. Private demands for public goods. American economic review 1973. 65 (3): 280-296

BOERCHERDING, T.E. and DEATON R.D. The demand for the services of non-federal governments. The American economic review. 1972. 62 (5):891-901.

BIFANI, Paolo. Medio ambiente y desarrollo sostenible. Instituto de Estudios Políticos para América Latina y Africa (IEPALA), 4ta. Edición, Madrid. 1999

BLOMQUIST; BERGER; HOEHN. New estimates of quality of life in urban areas. American economic review, 1988. núm. 78.

CABALLER Vicente et. al., El mercado inmobiliario urbano en España, Pirámide, Valencia, 2002.

CAMPOS, P., y Riera, P. Rentabilidad social de los bosques: Análisis aplicado a las dehesas y los montados ibéricos. Información Comercial Española, 1996. 751: 47-62.

CARTER, H. The study of Urban Geography. Arnold, Fourth edition. Great Britain. 1995

CASTELLS, Manuel. Reestructuración Económica, Revolución Tecnológica y Nueva Organización del Territorio. CEPAL, Documento CPRD-/96. Santiago de Chile. 1997

CLARK, David. Hedonic values of noxious activity: a a comparison of US worker responses by race and ethnicity. BRANDON P.S. et. al., (Edited). Evaluation of the Builtenvironment for sustainability. E \&FN SPON. 1997.

COASE, R. The problem of social cost. Journal of Law and Economics, 1960. 3:1-44. 
CONSTANZA, R. (ed.). Ecological Economics. The Science and Management of Sustainability, Columbia University Press, New York, USA. 1991.

CUMMINGS, R., y HARRISON, G.W. The measure and Decomposition of Nonuse Vlaues: A critical Review. Environmental and Resource Economics,1995. 5: 225-247.

DALY, H. (compilador). Economía, ecología, ética. Ensayos hacia una economía de estado estacionario. Fondo de Cultura Económica/ Economía Contemporánea, México. 1989.

DERYCKE, P. H. Economía y planificación urbana. Instituto de estudios de administración local. Madrid. 1983.

FITCH OSUNA, Jesús Manuel. Procedimiento para determinar el valor de la vivienda en el Área Metropolitana de Monterrey, Nuevo León, México: A partir del confort térmico. Tesis para obtener el Grado de Maestro en Valuación Inmobiliaria por la Universidad Autónoma de Nuevo León. México. 2002.

FUJITA, M.; KRUGMAN, P.; VENABLES, A. Encomia especial. Las ciudades, las regiones y el comercio internacional. Editorial Ariel. Barcelona. 2000.

FUSCO, L. Y NIJKAMP, P. Le Valutacioni per lo sviluppo sostenible de la città e del territorio. Franco Angeli. Milano. 1997.

GARCÍA Almirall, M. Pilar. La valoració Urbana sobre la base de les noves tecnologies de SIG. L'exemple de l'Hospitalet de Llobregat. Tesi Doctoral UPC (Inédita). 1998.

GARCÍA Almirall, Ma. Pilar. Un SIG para la evaluación ambiental de la ciudad. En "Territorial 2000. II Conferencia sobre Sistemas de Información Territorial". Pamplona, Noviembre. 2000. pág. 1.

GONZÁLEZ-PÁRAMO, J.M.; ONRUBIA, J. El gasto público en vivienda en España, Hacienda Pública Española, 1992. 120/121, págs. 189-217.

GRAVES, P. Migration and climate. Journal Science, 1980. num. 20.

GRILICHES, Z. Prices Indices and Quality Change, Harvard University Press, Cambridge, 1971.

HAIG, R.M. Toward an understanding of the Metropolis, Quaterly. Journal of Economics, 1926. 40.

HARTWICK, J.M. Intergenerational Equity and the Investing of Rents from Exhaustible resources. American Economic Review, 1977. 67: 972-974.

HAUSMAN, J.A. Contingent Valuation: A critical Assesment, North Holland, Holland. 1993.

HURD, R.M. Principles of city land values. The Record and Guide. New York. 1994.

JAEN GARCÍA, M. Y MOLINA MORALES, A. Modelos econométricos de tenencia y demanda de vivienda. Universidad de Almería. 1995

JOHANSSON, P.O. Valuing Environmental damage. Oxford Review of Economics Policy 1990. 6: $34-50$.

KNOX, P. Urban Social Geography. An Introducción. Nueva York. Longman. 1982. 
KRUMM, R. Neighbourhood amenities: an economic analysis. Journal of Urban Economics, 1960. number, 7.

LÓPEZ GARCíA, M. A. Algunos aspectos de la economía y la política de la vivienda, Investigaciones Económicas, 1992. vol. XVI,I, págs. 3-41.

McFADDEN D. AND LEONARRD. Issues in the Contingent Valuation of Environmental Goods: Methodologies for Data Collection and Analysis, en Contingent Valuation: A Critical Assessment, Hausman ed., 165-208. North-Holland. Amsterdam. 1993

MANNING, C. Intercity differences in home price appreciation, Journal of real estate Research, 1986. núm. 1.

MARSHALL, Alfred. Principios de economía. Ed. Aguilar. España. 1963.

MUTH, R. Cities and Hosing. Chicago UP, Chicago. 1969.

NARVAEZ TIJERINA, Adolfo. AEDIFICARE. Anuario de Investigaciones de la Facultad de Arquitectura de la Universidad Autónoma de Nuevo León. México. 2002.

NAREDO, J.M. y PARRA, F. (compiladores). Hacia una ciencia de los recursos naturales. Siglo Veintiuno de España Editores, Madrid, España. 1993.

NIJKAMP P. y PERRELS, A. Sustainable Cities in Europe, Earthscan. 1994.

PALMQUIST, R.B. Estimating the demand for the Characteristics of Housing. The Review of Economics and Statics, 1984. LXVI (3): 394-404.

PAPAGEORGIOU, G. J. On spatial consumer equilibriumo, Essays in Mathematical Land use Theory, Lexington Books, Lexington. 1976.

PEARCE, D. Economics values and the natural world. Earthscan, London, UK. 1993.

PEARCE, D.W, y TURNER, R.K. Economics of the Natural Resources and the Environment. Harvester Wheatsheaf, London, UK. 1990.

PEARCE, David; TURNER, Kerry. Economía de los recursos naturales y del medio ambiente, Colegio de Economistas de Madrid; Celeste Ediciones, España. 1995.

RICHARDSON, H. W. On the possibility of positive rent gradients, Journal of Urban Economies, 1977. 4.

RIERA, P. Manual de valoración contingente. Instituto de Estudios Fiscales, Madrid, España. 1994.

RIERA, P. Beneficio social del pasillo verde ferroviario de Madrid. Noesis, Madrid, España. 1995.

RIERA, P., DESCALZI, C. y RUIZ, A. El valor de los espacios de interés natural en España. Aplicación de los métodos de valoración contingente y coste de desplazamiento. Revista Española de Economía, número monográfico "Recursos Naturales y Medio Ambiente": 1994. Págs. 207-229. 
ROBACKS, J. Wages, rents and quality of life. Journal of political economy, 1982. núm. 90.

ROCA, J. Vers una interpretació espacial dels valors del sòl a Barcelona, tesi doctoral, Universitat de Barcelona. España. 1982.

ROCA , J. Los Precios del suelo en el ámbito metropolitano, Corporació Metropolitana de Barcelona. 1986.

ROCA CLADERA, J. La Estructura De Valores Urbanos: Un Análisis Teórico-Empírico. Ed. Instituto De Estudios De Administración Local. Madrid. 1988.

Rosen, Sherwin. Hedonic Prices and Implicit Markets: Product Differentiation in Pure Competition, Journal of Political Economy, 1974. 82(1), Jan.-Feb., pp. 34-55.

TIETENBERG, T. Environmental and Natural resource Economics. Harper Collins Publishers. New York. USA. 1988.

WINGO JR, Lowdon. Transportation and urban land. Washington D. C.: Resources for the Future, 1961.

YAMADA, H.: On the Theory of Residential Location: Accessibility, Space, Leisure and Environmental Quality, Papers ot the Regional Scence Association, 29, 1972. 